\title{
Optimierung eines Funksystems für hybride kaskadierte Netzwerke in der Fertigungsautomation
}

\author{
Lisa Underberg $^{1}$, Rüdiger Kays ${ }^{1}$, Steven Dietrich ${ }^{2}$ \\ ${ }^{1}$ Lehrstuhl für Kommunikationstechnik \\ Technische Universität Dortmund \\ \{lisa.underberg, ruediger.kays\}@tu-dortmund.de \\ ${ }^{2}$ DC-IA $/ \mathrm{EFC}$ \\ Bosch Rexroth AG \\ steven.dietrich@boschrexroth.de
}

\begin{abstract}
Zusammenfassung. Die Kommunikationsstrukturen industrieller Applikationen sind im Zuge von Industrie 4.0 einem Wandel unterworfen. Die aktuell verwendeten, kabelgebundenen Netzwerke sind einerseits hervorragend erprobt, bringen aber andererseits die typischen Nachteile mit sich. Diesbezüglich ist der Einsatz von drahtlosen Netzwerken aufgrund ihrer Flexibilität sowie ihrer einfachen Nachrüstbarkeit interessant. Diesen Vorteilen steht die bisher nur eingeschränkte Eignung insbesondere für die hohen Anforderungen industrieller Applikationen der Fertigungsautomation entgegen.

In diesem Beitrag werden Anforderungen von Applikationen der Fertigungsautomation zusammengefasst. Daraus wird deutlich, dass diese Anforderungen bisher nur von Industrial Ethernet Netzwerken (IEN) vollständig abgedeckt werden. Zur Unterstützung der Migration von IEN zu Funknetzwerken wird eine hybride, kaskadierte Topologie vorgestellt, in welcher ein bestehendes IEN durch eine drahtlose Technologie erweitert wird. Durch Analyse der Freiheitsgrade drahtloser Technologien wird herausgestellt, welche Eigenschaften ein Funksystem besitzen sollte, um sich für eine solche Erweiterung zu eignen.
\end{abstract}

\section{Einleitung}

Anwendungen der Fertigungsautomation (FA) stellen verglichen mit Anwendungen der Prozessautomation und der Zustandsüberwachung besonders strikte Anforderungen bezüglich Zuverlässigkeit und Latenz an ein Kommunikationssystem [Di17b,Un18]. Aus diesem Grund werden im Bereich der FA zurzeit kabelgebundene, Industrial Ethernet-basierte Netze (IEN) verwendet, deren Performanz bei hoher Datenrate zuverlässig und deterministisch vorhersehbar ist. Dennoch erfordern bereits einige aktuelle und vor allem künftige Anwendungen, die im Zuge von Industrie 4.0 verstärkt aufkommen werden, eine höhere Flexibilität und Mobilität des Kommunikationsnetzes als ein IEN bieten kann. Dementsprechend 
gewinnt die Verwendung von Funksystemen in der industriellen Kommunikation an Bedeutung [WSJ17].

Inzwischen decken spezialisierte industrielle Funksysteme wie WirelessHART und WSAN einige Teile industrieller Applikationen ab, insbesondere in der Prozessautomation und der Zustandsüberwachung. Lediglich im Bereich der typischen FA-Closed-Loop-Applikationen werden aufgrund der äußerst hohen Anforderungen weiterhin vorrangig IEN eingesetzt. Vor allem die kurze Zykluszeit von weniger als $1 \mathrm{~ms}$ und die hohe Zuverlässigkeit wird von aktuellen Funksystemen nicht erreicht. Um auch in diesem Bereich die Vorteile von Funksystemen nutzbar zu machen, entstehen kaskadierte Netze mit kabelgebundenen und kabellosen Netzwerkdomänen [SJB09,Ho16,Di17a]. Allerdings gibt es in hybriden Netzen mit einem IEN besondere Anforderungen an die Funktionalität des Gesamtnetzes. Diese Anforderungen werden in diesem Beitrag herausgestellt. Darauf aufbauend wird eine Empfehlung für das Design eines kabellosen Netzwerks für den Betrieb in einem hybriden, kaskadierten Netzwerk gegeben.

In Abschnitt 2 werden zunächst die Anforderungen von Applikationen der Fertigungsautomation zusammengefasst. Daraus werden vier zentrale Anforderungen an ein Kommunikationssystem für die Fertigungsautomation abgeleitet. Eine hybrides, kaskadiertes Netzwerk mit kabelgebundenen sowie drahtlosen Teilnetzwerken wird in Abschnitt 3 vorgestellt. Aktuell verfügbare Funksysteme werden hinsichtlich ihrer Eignung diskutiert. Das im Forschungsprojekt ParSec verwendete Parallel Sequence Spread Spectrum (PSSS)-Verfahren wird in Abschnitt 4 kurz eingeführt, welches aufgrund der Kombination von Code Division Multiple Access (CDMA) und Time Division Multiple Access (TDMA) dem MAC große Flexibilität bietet. Abschnitt 5 fasst die Ergebnisse zusammen und gibt einen kurzen Ausblick auf den Demonstrator, welcher im ParSec-Projekt realisiert wird. 


\section{Kommunikationssysteme in der Fertigungsautomation}

\subsection{Allgemeine Anforderungen von Applikationen der Fertigungsautomation}

Die Analyse von industriellen Applikationen hinsichtlich ihrer vielfältigen Anforderungen ist ein wichtiger Grundstein für die Leistungsbewertung von Kommunikationssystemen. Bereits 2007 wurden in Richtlinie 2185 Blatt 1 [VD07] Anforderungen verschiedener Applikationskategorien gegenübergestellt. Die Grenzen der Applikationskategorien wurden seitdem verfeinert, und auch die Kategorisierungsgrenzen selbst haben sich verschoben. Häufig wird die Kategorie der Fertigungsautomation - neben anderen - genannt und als die Kategorie mit den anspruchsvollsten Anforderungen hinsichtlich Zuverlässigkeit und Latenz beschrieben [Sc17,VD17].

Eine weiterführende Analyse zeigt, dass Applikationen selbst innerhalb der Fertigungsautomation so unterschiedliche Anforderungen haben, dass eine Verfeinerung in Unterkategorien sinnvoll ist. Die Aufteilung in Werkzeugmaschinen, Verpackungsmaschinen und Druckmaschinen ist inzwischen verbreitet [Fr14,Di17b]. Abbildung 1 zeigt die Anforderungen dieser drei Unterkategorien (vgl. [Di17b,Un18]).

Die Knoten einer Druckmaschine fordern eine hochgenaue Synchronisation mit einem Jitter der dezentralen Uhren von $<0,25 \mu$ s. Werkzeugmaschinen benötigen eine besonders kurze Zykluszeit von $0,5 \mathrm{~ms}$ bei gleichzeitig sehr geringer Fehlerrate. Verpackungsmaschinen haben vergleichsweise moderate Anforderungen.

\subsection{Zentrale Anforderungen der Fertigungsautomation an ein Kommunikationssystem}

Bisher werden die Anforderungen von Applikationen der Fertigungsautomation nur von Industrial Ethernet-basierten Netzen (IEN) vollständig abgedeckt. Durch die Analyse der Kerneigenschaften eines IENs werden in diesem Abschnitt allgemein gültige Anforderungen an ein Kommunikationsnetz abgeleitet. Bei der Betrachtung von typischen IENs wie Sercos III, EtherCAT und Profinet können folgende Gemeinsamkeiten abgeleitet werden:

1. IENs werden entsprechend ihres Zeitverhaltens kategorisiert:

- Keine Echtzeitfähigkeit

- Weiche Echtzeitfähigkeit

- Harte Echtzeitfähigkeit

- Isochrone Echtzeitfähigkeit

2. IENs sind meistens in einer logischen Sterntopologie mit einem zentralen Koordinator, dem IEN Master, und mehreren IEN Slaves aufgebaut

3. Die physikalische Topologie ist meist eine Linien- oder Doppelringtopologie

4. Der Austausch von zeitkritischen Daten (RT-Datenpakete) zwischen IEN Master und IEN Slaves findet zyklisch statt, zum Beispiel mit einer Zykluszeit von $1 \mathrm{~ms}$ 


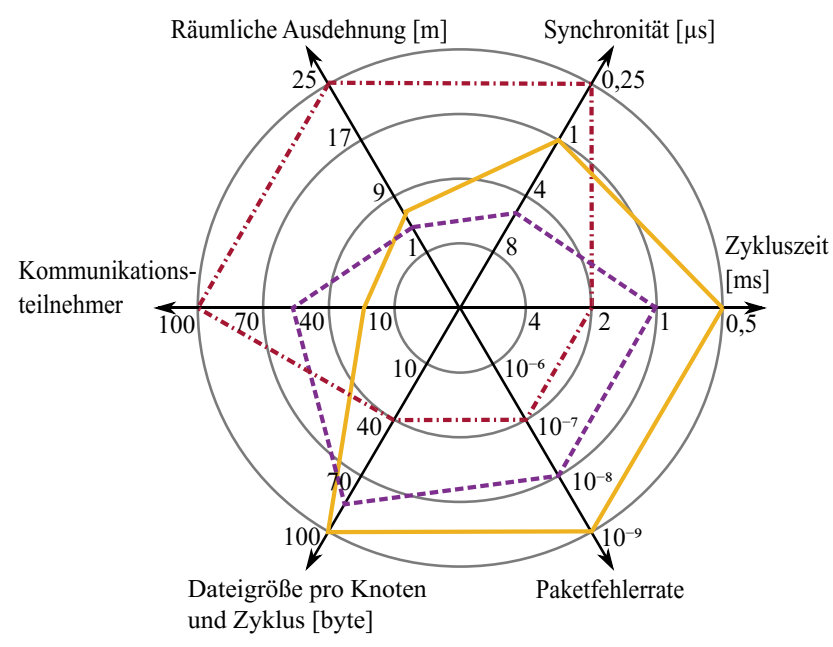

Werkzeugmaschinen -..-- Verpackungsmaschinen -.-.- Druckmaschinen

Abb. 1. Konsolidierte Anforderungen von drei Applikationsgruppen der Fertigungsautomation (vgl. [Di17a,Di17b]).

5. Alle Teilnehmer übernehmen Steuerdaten beziehungsweise aktualisieren Messwerte jeweils zum Global Sampling Point (GSP)

6. IENs sind durch ihre kabelgebundene Struktur weitgehend abhör- und angriffssicher

7. Aufgrund der kabelgebundenen Kommunikation werden IENs kaum durch Interferenzen gleicher oder anderer Systeme gestört

Aus diesen Gemeinsamkeiten können vier zentrale Anforderungen (zA) abgeleitet werden, die ein Kommunikationssystem - unabhängig von der zugrunde liegenden kabelgebundenen oder kabellosen Technologie - erfüllen muss:

zA1 Zuverlässige Übertragung der Applikationsdaten in jedem Zyklus

zA2 Hochgenaue Synchronisation aller Teilnehmer (Einhaltung des GSP)

zA3 Verschlüsselung und Authentifizierung der Kommunikation

zA4 Inter- und Intrasystemkoexistenz

Die Erfüllung von zA1 hängt wesentlich von der Bereitstellung einer ausreichenden Datenrate ab. Hier müssen natürlich nicht nur die Applikationsdaten selbst, sondern auch für die Übertragung nötiger Overhead wie die Zusatzinformation einer zyklischen Redundanzprüfung (Cyclic Redundancy Check, CRC) oder einer Vorwärtsfehlerkorrektur (Forward Error Correction, FEC) berücksichtigt werden.

zA2 wird erreicht, indem die dezentralen Uhren aller Teilnehmer hochgenau synchronisiert werden. Grundsätzlich gibt es zwei verschiedene Herangehensweisen zur Synchronisation verteilter Uhren. Im ersten Ansazt werden dedizierte 
Pakete versendet, die ausschließlich Informationen zum Uhrensynchronisation haben. Entsprechend wird Overhead generiert. Ein Beispiel dafür ist das Precision Time Protocol (PTP) [IE09]. Beim zweiten Ansatz hingegen werden die verteilten Uhren durch die physikalische Datenübertragung selbst synchronisiert. Dies ist möglich, da der GSP durch die Uhr des primären IEN Masters bestimmt wird, der gleichzeitig die Datenübertragung im Funksystem kontrolliert. Die Slaves des Funksystems berechnen den Zeitpunkt des GSPs daher auf einfache Weise relativ zur Detektion der PHY-Präambel. Bei diesem Ansatz wird ein minimaler Jitter zwischen Erzeugung eines IEN-Frames und Detektion des Pakets am Slave des Funksystems vorausgesetzt. Ebenfalls muss der Zugriffspunkt zwischen IEN und Funksystem geschaffen werden. Im Gegenzug ensteht durch dieses Vorgehen kein zusätzlicher Overhead.

Die Einhaltung von zA3 und zA4 ist in einem IEN inhärent durch das Übertragungsmedium gegeben. Die kabelgebundene Kommunikation kann nur intern mitgehört werden. Das Hinzufügen von unerkannten, nicht authentifizierten Teilnehmern ist durch die feste Struktur nur mit erheblichem Aufwnad möglich. Ebenfalls schützt die Abschirmung der Kabel vor Inter- oder IntraSystem-Interferenz. Natürlich ist die Einhaltung von zA3 und zA4 von ebenso großer Bedeutung bei der Verwendung von kabellosen Systemen. Die Datenübertragung muss verschlüsselt und authentifiziert werden, um das Eindringen von Angreifern zu vermeiden beziehungsweise zu detektieren. Ein effizientes Koexistenzmanagement ist notwendig, da im industriellen Umfeld drahtlose Systeme örtlich gleichzeitig mit Netzwerken bereits etablierter Technologien sowie weiteren Netzwerken der eigenen Technologie betrieben werden.

Dieser Beitrag fokussiert sich auf die Analyse von zA1 und zA2 und deren Einhaltung in einem hybriden Netzwerk mit kabelgebundenen sowie kabellosen Komponenten.

\section{Hybride, kaskadierte Netzwerke und ihre Eigenschaften}

Die Integration von kabellosen Netzwerken wird insbesondere in der Fertigungsautomation vorsichtig erfolgen. Hier sind die Applikationsanforderungen streng, sodass die Performanz eines Funksystems zunächst praktisch erpobt werden muss, bevor ein Einsatz infrage kommt. Gleichzeitig ist die Lebensdauer der Maschinen in diesem Feld länger als 10 Jahre [ET11], wodurch eine Nachrüstung bestehender Anlagen besonders wünschenswert ist.

Vor diesem Hintergrund sind hybride Netzwerke mit kabelgebundenen Industrial Ethernet-Komponenten sowie kabellosen Komponenten von Interesse. Sie erlauben die frühzeitige Integration von Funksystemen in Anwendungen der Fertigungsautomation, wodurch deren Erprobung unter realen Bedingungen ermöglicht wird. Aus Entwicklersicht bietet dies die Möglichkeit, die Systeme mit direktem Bezug zur Anwendung weiterzuentwickeln. Gleichzeitig wird die Akzeptanz von Funksystemen gefördert. Durch diesen Prozess wird die Integration von und später vollständige Migration zu kabellosen Kommunikationsnetzen begünstigt. 


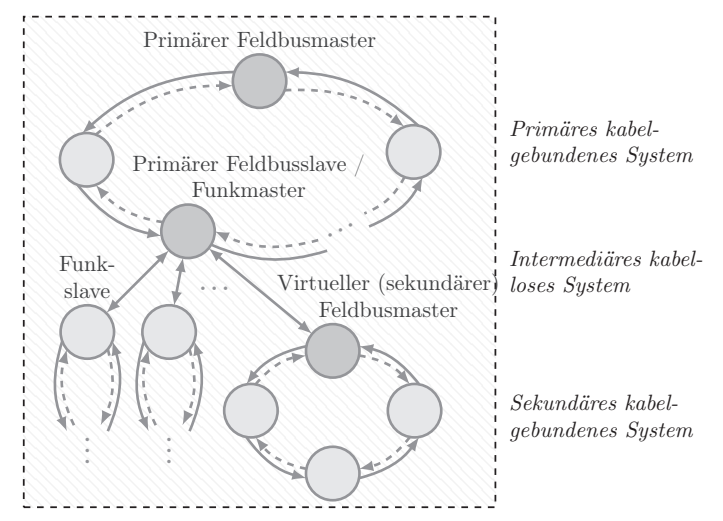

Abb. 2. Hybrides Netzwerk aus IEN mit drahtloser Erweiterung (vgl. [Un18]).

Abbildung 2 zeigt eine generische Topologie eines solchen hybriden Kommunikationssystemes anhand eines primären und eines sekundären, kabelgebundenen Netzwerks. Grundsätzlich kann die Kaskadierung fortgesetzt werden. Das primäre IEN ist in einer typischen Doppelringtopologie abgebildet. Einer der Teilnehmer im primären IEN übernimmt gleichzeitig die Rolle des Masters der intermediären drahtlosen Netzwerks. Der Teilnehmer kann sowohl ein primärer IEN Slave sowie der primäre IEN Master sein. Je nach konkretem Anwendungsfall kann die Ende-zu-Ende-Latenz reduziert werden, indem der IEN Master gleichzeitig das Funksystem kontrolliert.

Das intermediäre Funksystem ist in einer einfachen Sterntopologie angeordnet, da diese die Realisierung einer deterministischen Latenz vereinfacht. Die Slaves des Funksystems sind gleichzeitig Master der sekundären IENs. Die sekundären IEN Master emulieren jeweils die Funktion des primären IEN Masters, wodurch das Funksystem aus Sicht der sekundären IEN Slaves transparent wird. Ebenfalls emuliert der Teilnehmer des primären IENs, der gleichzeitig der Master des Funksystems ist, die sekundären IEN Slaves. So wirkt das Funksystem auch aus Sicht des primären IEN Masters transparent, sofern zA1 und zA2 eingehalten werden. Zur Sicherung der drahtlosen Kommunikation müssen zusätzlich zA3 und zA4 erfüllt sein.

\subsection{Verwendbarkeit von bereits etablierten drahtlosen Technologien}

Aktuell sind vielfältige drahtlose Technologien verfügbar. Sie reichen von Ultra Wideband (UWB)-Ansätzen, die eine gute Koexistenz mit bestehenden Technologien versprechen, bis hin zu neuen Ansätzen aus dem Bereich 5G oder IEEE 802.11ax. Unabhängig von einer bestimmten Technologie müssen die vier zentralen Anforderungen der Fertigungsautomation erfüllt werden, damit ein Kommunikationssystem sich für den Einsatz in der Fertigungsautomation eignet. 
Technologien, die auf IEEE 802.15.4 [IE15] basieren, sind beispielsweise ZigBee [Zi12] und WirelessHART [(I10]. Diese eignen sich aufgrund ihrer Mesh- oder Cluster Tree-Topologie für Anwendungen der Prozessautomation. Werden sie in ihrer als optional definierten Sterntopologie verwendet, so können sie bestimmte Anwendungen der FA mit vergleichsweise lockeren Anforderungen hinsichtlich zA1 und zA2 erfüllen. WirelessHART unterstützt nur eine Meshtopologie, in der die Uhrensynchronisation aus zA2 erschwert ist.

Wireless Sensor Actuator Network (WSAN) [PN12] und Bluetooth [Bl14] verwenden ein Frequenzspreizverfahren (Frequency Hopping Spread Spectrum, FHSS) wie in IEEE 802.15.1 [IE05] standardisiert. Die hier ermöglichte Datenrate von bis zu $3 \mathrm{Mbit} / \mathrm{s}$ ist allerdings zu klein für die meisten Anwendungen der Fertigungsautomation, wodurch die Einhaltung von zA1 fraglich ist.

\subsection{Freiheitsgrade beim Entwurf eines Funksystems}

Wie in Abschnitt 3.1 kurz diskutiert, eignen sich aktuell verfügbare, drahtlose Technologien nur für Teilbereiche der Fertigungsautomation. Insbesondere die Einhaltung von zA1 und zA2 ist nicht gleichzeitig gegeben. Daraus folgt, dass diese Aspekte bei der Entwicklung neuer, drahtloser Ansätze schon beim grundlegenden Design berücksichtigt werden müssen.

Beim grundlegenden Design sind vier Entscheidungen zu treffen, welche in Abb. 3 zusammengefasst sind. Im Folgenden wird die Eignung der verschiedenen Optionen hinsichtlich zA1 und zA2 bewertet.

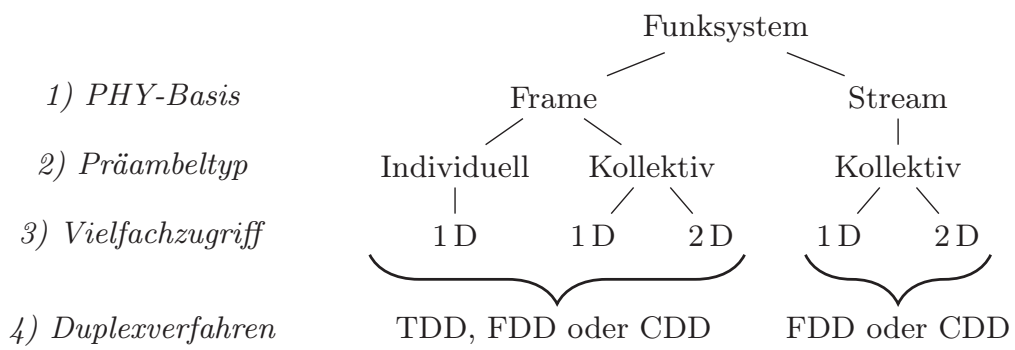

Abb. 3. Entscheidungsbaum zum optimierten Entwurf für ein intermediäres Funksystem.

Die erste Option betrifft die PHY-Basis. Die Übertragung der Daten kann entweder in eigenständigen PHY-Frames organisiert sein oder als kontinuierlicher Stream von PHY-Symbolen realisiert werden. Bei einer frame-basierten Realisierung sind zwei PHY-Frames durch einen Interframe Space (IFS) getrennt. Jeder PHY-Frame beginnt mit einer Präambel, auf welche PHY-Symbole zur Nutzdatenübertragung folgen. Die Framelänge ist dabei durch die Kohärenzzeit der Kanals begrenzt. Bei einer stream-basierten Realisierung wird die Übertragung nicht pausiert, sondern erfolgt unterbrechungslos ohne IFS. Regelmäßig werden 
einige PHY-Symbole für die Übertragung einer Präambel genutzt, die zur Aufrechterhaltung der Synchronisation zwingend benötigt wird. Hier muss die Dauer zwischen zwei Präambeln kleiner als die Kohärenzzeit sein.

Die Wahl des Präambeltyps ist die zweite Option. Bei einer durch jeden Teilnehmer individuell genutzten Präambel werden in jedem PHY-Frame ausschließlich die Daten einer bestimmten Ende-zu-Ende-Verbindung übertragen. Wird eine kollektive Präambel verwendet, so können die nachfolgenden PHYSymbole die Daten verschiedener Verbindungen enthalten. Bei einer streambasierten PHY-Basis kann nur eine kollektive Präambel verwendet werden, da eine individuelle Datenübertragung gar nicht erst ermöglicht wird. Eine kollektive Präambel ermöglicht die flexible Aufteilung der Übertragungsressourcen in zwei Domänen, sodass diese möglichst effizient genutzt werden können. Framebasierte Übertragungssysteme mit individueller Präambel sind beispielsweise aus IEEE 802.15.1 oder IEEE 802.15.4 bekannt. Eine kollektive Präambel bei framebasiertem PHY wird aktuell in IEEE 802.11ax oder im 5G-Kontext diskutiert.

Nach der Wahl des Präambeltyps wird festgelegt, ob der Vielfachzugriff (Multiple Access) in einer oder in zwei Domänen ermöglicht wird. Typische Vielfachzugriffsverfahren sind Time Divison Multiple Access (TDMA), Frequency Division Multiple Access (FDMA) sowie Code Division Multiple Access (CDMA). Bei einer individuellen Präambel werden die Übertragungsressourcen nur in einer Domäne (1D) - Zeit, Frequenz oder Code - aufgeteilt. Wird eine kollektive Präambel verwendet, so kann TDMA mit FDMA beziehungsweise CDMA kombiniert werden. Die Übertragungsressourcen werden dann in Zeit und Frequenz (TDMA + FDMA) oder in Zeit und Code (TDMA + CDMA) aufgeteilt. Die Kombination von TDMA und FDMA ist von OFDMA bekannt, während beispielsweise ein PSSS-System TDMA und CDMA kombiniert.

Die vierte Option betrifft das Duplexing. In einer Sterntopologie bezieht sich das Duplexing auf die Realisierung der bidirektionalen Kommunikation in Downund Uplink. In vielen Systemen wird eine zeitliche Trennung - Time Division Duplex (TDD) - gewählt. Alternativ dazu kann ein Frequency Division Duplex (FDD) oder Code Division Duplex (CDD) genutzt werden. Für den Betrieb eines FDD-Systems muss genug Bandbreite zur Verfügung stehen, während ein CDDSystem eine Trennung zwischen RF-Uplink und RF-Downlink erreichen muss.

\subsection{Optimierter Entwurf des Funksystems}

Hinsichtlich der für zA1 geforderten Datenrate bietet der stream-basierte PHY einen Vorteil, da hier durch die ununterbrochene Übertragung die verfügbaren Ressourcen vollständig genutzt werden. Leider kann die Uhrensynchronisation kaum über die PHY-Übertragung selbst durchgeführt werden. Eine Ausnahme gibt es, wenn das GSP-Intervall ein exaktes Vielfaches der PHY-Symboldauer ist. Es muss also ein Synchronisationsverfahren verwendet werden, dessen zusätzlicher Overhead den Vorteil der ununterbrochenen Übertragung nicht vollständig kompensiert.

Dementsprechend ist ein frame-basierter PHY hinsichtlich zA2 vielversprechender, da hier die Uhrensynchronisation an den jeweiligen Beginn eines PHY- 
Frames gekoppelt werden kann. Dieser Ansatz setzt natürlich voraus, dass alle Verzögerungszeiten, wie sie zum Beispiel durch die Verarbeitung entstehen, in allen Teilnetzwerken bekannt sind. Im Bereich der Fertigungsautomation ist diese Annahme allerdings sinnvoll, da die Kommunikationsstruktur eines IENs unveränderlich ist, sobald der Betrieb aufgenommen wird. Lediglich die Signallaufzeit ist unbekannt. Bei einer räumlichen Ausdehnung von maximal $25 \mathrm{~m}$ hat diese mit $<0,1 \mu$ s einen vernachlässigbaren Einfluss auf die Genauigkeit der GSPSynchronisation.

Beim frame-basierten PHY besteht nun die Wahl zwischen einer individuellen und einer kollektiven Präambel. Da der Overhead bei Nutzung einer individuellen Präambel größer als bei einer kollektiven Präambel ist, ist letztere hinsichtlich der Einhaltung von zA1 vorzuziehen. Ebenso ist ein FDD-Ansatz wünschenswert, sofern die nötigen Frequenzbänder zur Verfügung stehen. Für ein TDD-Verfahren wird eine entsprechend höhere Datenrate benötigt.

\section{Das Projekt ParSec: Paralleles und sicheres Funksystem}

Ein Funksystem, welches den vorangegangenen Überlegungen in Abschnitt 3.3 entspricht, wird im Projekt ParSec [BMb,Kr16] entwickelt. ParSec ist eines der acht Projekte der BMBF-Initiative „Zuverlässige drahtlose Kommunikation für die Industrie "(ZDKI) [BMa].

Das in ParSec entworfene Funksystem basiert auf einem Parallel Sequence Spread Spectrum (PSSS)-Ansatz [Wo04,SW04]. Das ParSec Funksystem wird zur Synchronisation der dezentralen Uhren der Teilnehmer frame-basiert realisiert. Zur Reduktion des Overheads wird eine kollektive Präambel gewählt. PSSS erlaubt die Kombination von TDMA und CDMA und ist damit hinsichtlich der Ressourcenaufteilung ähnlich flexibel wie ein auf einem OFDM-Ansatz basierendes System. Das Duplexing erfolgt via FDD.

Abbildung 4 zeigt schematisch die Ressourcenaufteilung im ParSec Demonstrator. Entsprechend der Analyse in [Di18] wird eine sequentielle Aufteilung gewählt, da sich diese für die Erreichung kurzer Latenzen besonders gut eignet.

Im Rahmen des Projekts ParSec, das bis zum 31.12.2018 läuft, entsteht ein Demonstrator eines kaskadierten hybriden Netzes, mit dem die Ansätze darüber hinaus praktisch erprobt werden. In ParSec wurde als typisches IEN Sercos III ausgewählt. Aktuell wurde die Funktionalität der Sercos III-ParSec-Schnittstelle mittels eines PHY-Emulators nachgewiesen. Die Integration des in ParSec implementierten PSSS-Funksystems, dessen angestrebte Parametrierung in Tabelle 1 zusammengefasst wird, ist bis zum Ende der Projektlaufzeit geplant. 


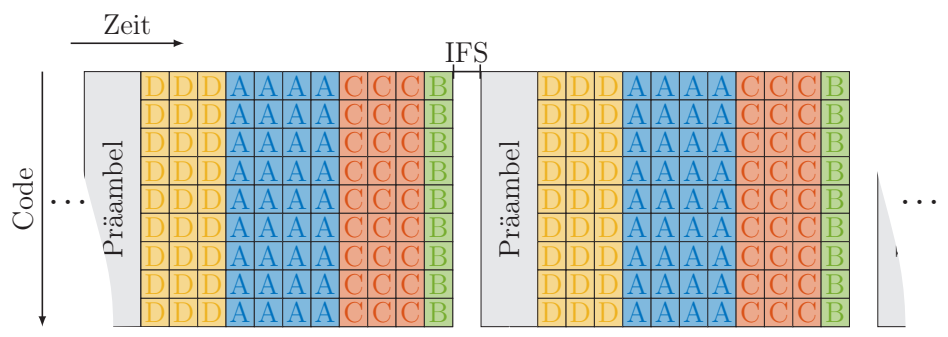

Abb. 4. Frame-basierter PHY mit 2 D Ressourcenaufteilung und gemeinsamer Präambel im PSSS Frame.

Tabelle 1. Zielparameter des ParSec Funksystems für den ParSec Demonstrators.

\begin{tabular}{lc}
\hline Mittenfrequenz & $5,8 \mathrm{GHz}$ \\
m-Sequenz für PSSS & $m=255 \mathrm{Chips}$ \\
Chipfrequenz & $20 \mathrm{MHz}$ \\
Cos-Rolloff-Filter & 0,2 \\
Bandbreite & $24 \mathrm{MHz}$ \\
APP-Zykluszeit & $1 \mathrm{~ms}$ \\
PHY-Datenrate & $17,34 \mathrm{Mbit} / \mathrm{s}(68 \mathrm{PSSS} \mathrm{Symbole} / \mathrm{ms})$ \\
MAC-Datenrate & $12,75 \mathrm{Mbit} / \mathrm{s}(50 \mathrm{PSSS}$ Symbole $/ \mathrm{ms})$ \\
FEC & BCH $(255,131,18) \mathrm{mit}$ Coderate $\approx 0,5$ \\
Authentifizierung & CMAC: 64 Bit $/$ Datenpaket \\
Verschlüsselung & Prince Cipher mit 64 Bit Blocklänge \\
APP-Datenrate & 48 Datenpakete $/$ ms \\
\hline
\end{tabular}

\section{Zusammenfassung und Ausblick}

In diesem Beitrag wurden Industrial Ethernet-basierten Netze (IEN) hinsichtlich ihrer Gemeinsamkeiten analysiert und vier zentrale Anforderungen (zA) an ein Kommunikationssystem im Bereich der Fertigungsautomation identifiziert. Diese vier Anforderungen müssen für einen reibungslosen Betrieb im Bereich der Fertigungsautomation erfüllt werden. Insbesondere für ein hybrides Netzwerk mit kabelgebundenen sowie drahtlosen Netzwerkdomänen sind diese Anforderungen herausfordernd. Zum Beispiel muss im gesamten Netzwerk neben der zuverlässigen und schnellen Übertragung der Applikationsdaten eine genaue Synchronisation dezentraler Uhren gewährleistet werden. Nur so kann der Global Sampling Point (GSP), der im IEN für Closed-Loop-Applikationen benötigt wird, ausreichend präzise eingehalten werden. Darüber hinaus sind Verschlüsselung und Authentifizierung der Kommunikation sowie die Koexistenz des Übertragungssystems mit gleichen und unterschiedlichen Übertragungssystemen zu berücksichtigen.

Dieser Beitrag zeigte, dass aktuell verfügbare Funksysteme diese vier zentralen Anforderungen noch nicht erfüllen, da bereits die unteren Kommunikations- 
schichten, physikalische Schicht (PHY) und Medienzugriff (MAC), ungeeignet sind. Beispielsweise führt ein CSMA/CA-Verfahren im PHY zu einer nicht deterministischen Latenz. Dies kann auf höheren Kommunikationsschichten nicht mehr kompensiert werden. Um eine als intermediäres Funknetzwerk geeignete drahtlose Technologie zu entwerfen, müssen die Anforderungen also bereits bei PHY- und MAC-Design berücksichtigt werden.

Die grundlegenden Optionen beim Design einer drahtlosen Technologie wurden daher in diesem Beitrag zusammengefasst. Die Wahl der PHY-Basis, des Präambeltyps, des Vielfachzugriffs sowie der Duplexing-Strategie wurde hinsichtlich der zentralen Anforderungen diskutiert. Hinsichtlich der zuverlässigen Übertragung der zyklischen Applikationsdaten (zA1) und der hochgenauen Synchronisation der dezentralen Uhren aller Teilnehmer (zA2) wurde ein framebasierter PHY mit kollektiver Präambel und 2 D Vielfachzugriff empfohlen. Je nach erreichter Datenrate kommen für das Duplexing im aktuellen Stand der Technik TDD oder FDD infrage.

Ein solches, optimiertes Funksystem wird im Projekt ParSec realisiert. Der auf PSSS basierende PHY wurde entsprechend der Anforderungen von ClosedLoop-Applikationen parametriert. Innerhalb der Laufzeit bis Dezember 2018 wird ein Demonstrator realisiert, der die Kopplung des ParSec Funksystems mit einem IEN zeigt. In ParSec wurde Sercos III ausgewählt. Aktuell wurde die Funktionalität der Sercos III-ParSec-Schnittstelle mittels eines PHY-Emulators nachgewiesen. Die Integration des in ParSec implementierten PSSS-Funksystems ist bis zum Ende der Projektlaufzeit geplant.

\section{Hinweis}

Das diesem Bericht zugrunde liegende Vorhaben wurde mit Mitteln des Bundesministeriums für Bildung und Forschung unter den Förderkennzeichen 16KIS0223 und 16KIS0225 gefördert. Die Verantwortung für den Inhalt liegt beim Autor.

\section{Literatur}

[Bl14] Bluetooth SIG: Bluetooth Specification Version 4.2. S. 2772, 2014.

[BMa] BMBF Initiative „Zuverlässige drahtlose Kommunikation in der Industrie ". www.industrialradio.de.

[BMb] BMBF Projekt ParSec - Ein paralleles zuverlässiges und sicheres Funksystem zur latenzoptimierten Fabrikautomatisierung. www.parsec-projekt.de.

[Di17a] Dietrich, Steven; May, Gunther; v. Hoyningen-Huene, Johannes; Müller, Andreas; Fohler, Gerhard: Anforderungsanalyse und Optimierungen kaskadierter Netzwerke für die Fertigungsautomatisierung. In: 2017 8. Jahreskolloquium "Kommunikation in der Automation"(KommA). November 2017.

[Di17b] Dietrich, Steven; May, Gunther; Wetter, Oliver; Heeren, Holger; Fohler, Gerhard: Performance Indicators and Use Case Analysis for Wireless Networks in Factory Automation. In: 2017 IEEE 22nd International Conference on Emerging Technologies and Factory Automation (ETFA). September 2017. 
[Di18] Dietrich, S.; Underberg, L.; May, G.; Kays, R.; Fohler, G.: Optimized resource allocation for cascaded communication networks in factory automation. In: ICIT 2018; 19th International Conference on Industrial Technology. Feb 2018.

[ET11] ETSI: TR 102 889-2 V1.1.1, Electromagnetic compatibility and Radio spectrum Matters (ERM); System Reference Document; Short Range Devices (SRD); Part 2: Technical characteristics for SRD equipment for wireless industrial applications using technologies different from Ultra-Wide Band (UWB). Bericht, European Telecommunications Standards Institute, 2011.

[Fr14] Frotzscher, Andreas; Wetzker, Ulf; Bauer, Matthias; Rentschler, Markus; Beyer, Matthias; Elspass, Stefan; Klessig, Henrik: Requirements and current solutions of wireless communication in industrial automation. In: Communications Workshops (ICC), 2014 IEEE International Conference on. IEEE, S. 67-72, 2014.

[Ho16] von Hoyningen-Huene, Johannes; Mueller, Andreas; Dietrich, Steven; May, Gunther: Comparison of wireless gateway concepts for industrial real-timecommunication. In: IEEE International Conference on Emerging Technologies and Factory Automation (ETFA'16). IEEE, S. 1-4, 2016.

[(I10] (IEC), International Electrotechnical Commission: , IEC 62591 Ed. 1.0: Industrial commun. networks - wireless commun. network and commun. profiles WirelessHART, 2010.

[IE05] IEEE: Standard 802.15.1-2005, IEEE standard for information technology local and metropolitan area networks - specific requirements - Part 15.1a: Wireless medium access control (MAC) and physical layer (PHY) specifications for wireless personal area networks (WPAN). S. 700, 2005.

[IE09] IEEE: , IEEE Standard for a precision clock synchronization protocol for networked measurement and control systems, Feb 2009.

[IE15] IEEE: Standard for low-rate wireless networks. Standard 802.15.4-2015 (Revision of IEEE Std 802.15.4-2011), 2015.

[Kr16] Kraemer, Rolf; Methfessel, Michael; Kays, Rüdiger; Underberg, Lisa; Wolf, A.C.: ParSec: A PSSS approach to industrial radio with very low and very flexible cycle timing. In: EUSIPCO 2016; 24th European Signal Processing Conference. 2016.

[PN12] PNO: WSAN air interface specification technical specification, Version 1.0. S. 71, 2012.

[Sc17] Schulz, Philipp et al.: Latency Critical IoT Applications in 5G: Perspective on the Design of Radio Interface and Network Architecture. IEEE Communications Magazine, 55(2), 2017.

[SJB09] Sauter, Thilo; Jasperneite, Jürgen; Bello, Lucia Lo: Towards New Hybrid Networks for Industrial Automation. In: ETFA. Jgg. 9, S. 1141-1148, 2009.

[SW04] Schwetlick, H.; Wolf, A.: PSSS - Parallel Sequence Spread Spectrum a Physical Layer for RF Communication. In: Consumer Electronics, 2004 IEEE International Symposium on. S. 262-265, Sept 2004.

[Un18] Underberg, L.; Dietrich, S.; ; Kays, R.; Fohler, G.: Towards hybrid wiredwireless networks in industrial applications. In: IEEE International Conference on Industrial Cyber Physical Systems. Mai 2018.

[VD07] VDI/VDE: VDI/VDE Richtlinie 2185: Funkgestuetzte Kommunikation in der Automatisierungstechnik. Bericht, Verein Deutscher Ingenieure, Verband der Elektrotechnik Elektronik Informationstechnik, 2007.

[VD17] VDE ITG AG Funktechnologie 4.0: Funktechnologien für Industrie 4.0. Bericht, Verband der Elektrotechnik Elektronik und Informationstechnik, 2017. 
[Wo04] Wolf, A.: , Verfahren zum Uebertragen eines Datenworts, Patent DE 10301 250 A1, Jul. 29, 2004.

[WSJ17] Wollschlaeger, M.; Sauter, T.; Jasperneite, J.: The future of industrial communication: Automation networks in the era of the Internet of Things and Industry 4.0. IEEE Industrial Electronics Magazine, 11(1):17-27, March 2017.

[Zi12] ZigBee Alliance: ZigBee Specification, Document 053474r20. S. 594, 2012.

Open Access Dieses Kapitel wird unter der Creative Commons Namensnennung 4.0 International Lizenz (http://creativecommons.org/licenses/by/4.0/deed.de) veröffentlicht, welche die Nutzung, Vervielfältigung, Bearbeitung, Verbreitung und Wiedergabe in jeglichem Medium und Format erlaubt, sofern Sie den/die ursprünglichen Autor(en) und die Quelle ordnungsgemäß nennen, einen Link zur Creative Commons Lizenz beifügen und angeben, ob Änderungen vorgenommen wurden.

Die in diesem Kapitel enthaltenen Bilder und sonstiges Drittmaterial unterliegen ebenfalls der genannten Creative Commons Lizenz, sofern sich aus der Abbildungslegende nichts anderes ergibt. Sofern das betreffende Material nicht unter der genannten Creative Commons Lizenz steht und die betreffende Handlung nicht nach gesetzlichen Vorschriften erlaubt ist, ist für die oben aufgeführten Weiterverwendungen des Materials die Einwilligung des jeweiligen Rechteinhabers einzuholen. 